\title{
26
}

\section{Enhancing ATM Network Performance by Optimizing the Virtual Network Configuration}

\author{
A. Faragó ${ }^{2}$, S. Blaabjerg ${ }^{1}$, W. Holender ${ }^{1}$, B. Stavenow ${ }^{1}$, \\ T. Henk ${ }^{2}$, L. Ast ${ }^{2}$, S. Székely ${ }^{2}$ \\ ${ }^{1}$ Ellemtel Telecommunications Systems Laboratories \\ S-22370 Lund, Sweden \\ ${ }^{2}$ Dept. of Telecommunications and Telematics \\ Technical University of Budapest \\ XI. Sztoczek u. 2, Budapest, Hungary H-1111 \\ Phone: +3614631861 \\ Fax: +361463 3107 \\ E-mail: faragoettt-atm.ttt.bme.hu
}

\begin{abstract}
Virtual or logical subnetworks are expected to play an important role in large B-ISDN configurations. This gives an additional degree of freedom to ATM network architectures, since even for a fixed physical network the logical configuration can still vary depending on particular demands and conditions. This new degree of freedom calls for new solutions to utilize the opportunity for the potential enhancement of network performance by optimizing the logical configuration, as part of optimizing the distributed network architecture. In this paper a framework and model, along with efficient solution algorithms, are presented to dimension virtual ATM networks on top of the same physical infrastructure network, such that the virtual networks share the infrastructure, while the total network revenue is optimized. The algorithms are tried on various network scenarios and a trade-off between the quality of the result and running time is exhibited.
\end{abstract}

Keywords: ATM network, virtual subnetwork, network partitioning, network revenue. 


\section{Introduction}

The trend towards service integration in telecommunications has been steadily more profound and at the time when ATM was introduced the expectations regarding the range of services that could be integrated had practically no limitations. During the last few years, however, it has been recognized that it is not at all easy to integrate services with very different demands regarding e.g. bandwidth, grade of service (GoS) or congestion control functions. The latest development within the ITU SG13 with the agreement of the definitions of 4 ATM bearer capabilities clearly demonstrate this. In some cases it turns out to be better to support different services by offering separate logical networks, and limiting the degree of integration to only partial, rather than complete, sharing of physical transmission and switching resources.

A second context in which separation into logical networks may take place is virtual leased networks. Large business customers realizing e.g. LAN interconnections may require guaranteed resources. Furthermore, virtual paths are special cases of logical networks and peak rate allocation of VP's can be seen as a (virtual) separation of resources.

Resource separation for segregation of ATM layer bearer capabilities, for offering different GoS classes, virtual leased networks with guaranteed resources and peak rate allocated virtual paths are examples of a new feature in the design, dimensioning and management of ATM networks. On top of the physical infrastructure a number of logical or virtual subnetworks can co-exist, sharing the same physical transmission and switching capacities.

The complete partitioning of the infrastructure network is an important component in network optimization and can be seen as the contrast to complete sharing. It is evident that in many circumstances a solution somewhere between the two extremes with a minimum guaranteed resource and possibility to use a certain number of extra resources will be applied. However, since complete partitioning is an important landmark in the context and since so far most work has been done only for isolated links, an investigation of the issue on the network level is of importance.

The problems with full service integration are also realized in [12], and an optimization model taking into account transmission, switching and set up cost is developed in which different strategies like complete partitioning, complete sharing and sharing with trunk reservation can be evaluated. In [1] a greedy algorithm for Dynamic Capacity Management is used to design 
VP networks and the problem of gathering reliable measurement data is also considered. Concerning multi-rate models, [13] gives an excellent overview.

The objective of this paper is to formulate an optimization model of the partitioning problem for a network and to present three efficient algorithms that solve the problem. The objective function is the total network revenue, and together with the physical constraints also grade of service constraints are taken into account in the simplest model.

In Section 2 the B-ISDN network environment in which the model is to be applied is described and further motivation for and consequence of the partitioning approach is given. Section 3 presents the optimization model, while Section 4 describes three algorithms that find solutions.

\section{B-ISDN and Network Partitioning}

In this section the B-ISDN scenario considers a situation that is somewhat more developed than it is today.

\section{$2.1 \quad$ B-ISDN and Overlay Networks}

A fully developed B-ISDN will have a very complex structure with a number of overlay networks. Conceptual tools to simplify the description are needed and one such conceptual model suitable of describing overlay networks is the Stratified Reference Model, [7]. This model adopts the general layering of the OSI model, it works with the three lowest layers, but allows for recursion by a generalization of the physical layer.

Adopting the concept of the Stratified Reference Model, the B-ISDN will consist of the following strata, see Fig. 1. A transmission stratum based on $\mathrm{SDH}$ at the bottom, a cross connect stratum based on either SDH or ATM on top of that, which acts as an infrastructure for the ATM VP/VC stratum with switched connections. Finally, the large set of possible applications uses the ATM or cross connect stratum as an infrastructure.

\subsection{Cross Connect and Partitioning}

In this paper the focus will be on the ATM and the cross connect stratum in general and on partitioning of the ATM stratum network in particular. 
Whether the cross connect stratum is realized by SDH or ATM will have important implications for the partitioning, see Fig. 2. If the cross connect stratum is based on SDH and the ATM network is realizing different QoS classes by resource separation, the partitioning can only be done in integer portions of the STM modules of the SDH structure, see Fig. 2.a. On the other hand, if the cross connect is realized by ATM virtual paths (VP), then no integrality restriction exists and the partitioning can be done in any real portions, see Fig 2.b. The SDH cross connect solution will therefore give rise to a model that is discrete in the ATM link capacities while the ATM cross connect solution gives rise to a continuous model. It should be emphasized that if the partitioning is made in order to support different QoS classes, then either the integer solution should be adopted or the ATM switches will have to be designed in a way to support partitioning at the individual input and output ports.

\subsection{Relevant QoS Parameters}

Since ATM has similarities with both packet switched and circuit switched networks it is not a priori obvious which properties should have the greatest impact to an optimization model. However, it is the data transfer phase when the similarities with packet switched networks are the largest. At the connection setup phase the similarities to circuit switching dominate, especially if a preventive connection control concept with small ATM switch buffers has been adopted together with the equivalent bandwidth concept.

In an optimization model that models the call scale phenomenon it is natural to take the view that an ATM network can be modeled as a multirate circuit switched network in which the most important quality of service parameter is the connection blocking probability. This is the basis for the model to be considered in the next section.

\section{The Optimization Model}

A fixed infrastructure network with a number of nodes and a number of trunk groups connecting the nodes in an arbitrary way is considered. This network will model the cross connect stratum in the B-ISDN overlay network.

On top of this network a number of logically separated ATM networks are to be carried. The topology of these virtual or logical ATM networks will, 
in general, differ from the topology of the underlying network and an ATM link may use more than one trunk group. An ATM network can be a virtual leased network for a large business customer, it can be the part of an overall ATM network realizing a QoS class by resource separation, or it can simply be a virtual path.

The first set of constraints, expressible by linear inequalities in the model, comes from the natural fact that the sum of ATM link capacities on a physical trunk group cannot exceed the capacity of the trunk group.

The traffic demand between any two nodes in any of the ATM networks is assumed given, and a number of fixed routes in each of the ATM networks is also assumed given in advance. In general, there exists a number of routes between a given node pair. One objective of the optimization model is to distribute the traffic offered to the node pair optimally between the routes which can realize the communication. The distribution of the offered traffic between the possible routes is termed load sharing and the parameters according to which it takes place are called load sharing coefficients.

A second set of constraints in the model comes from the fact that the traffic demand between any pair of nodes should be equal to the sum of traffic demands on the routes realizing communication between the origindestination pair. This means, the sum of load sharing coefficients is one.

To explain the idea in the simplest case, each virtual ATM network is assumed to operate under fixed non-alternate routing. A connection attempt is assigned at random to a single route, and if insufficient resources are available, the connection request is blocked and disappears from the system. Since alternate routing complicates the analysis, we restrict ourselves to the simplest fixed routing case in this paper.

The bandwidth demand of any ATM connection is characterized by a single parameter that can be interpreted either as equivalent bandwidth in case of adoption of the sustainable cell rate, or as peak rate of the connection in case of peak rate allocation. It is assumed that the one-parameter characterization of the ATM connections together with an adequate admission control algorithm ensures that cell level GoS degradations, like cell loss, cell delay and cell delay variation are well under control, and need not be considered at this level. Instead, the main GoS parameter in this context is the call scale parameter: connection blocking probability.

For reasons of fairness it is natural to impose a third set of constraints, namely the GoS constraint, that the connection blocking probability on each 
route should not exceed a given maximum value.

The objective function is the total network revenue summed up over all virtual ATM networks. It is assumed that each accepted connection will generate revenue at a given rate depending on the traffic type and route, and the total network revenue can then be seen as a weighted sum of carried traffic values. For a complete mathematical formulation see [2].

To summarize, the task of the optimization model is to find the partitioning of the infrastructure network into virtual subnetworks and to find the load sharing coefficients for each ATM node pair which maximizes the total network revenue subject to the physical constraints listed above and optionally also the GoS constraints.

\section{Solution Approaches}

The objective function to be optimized is inherently difficult to deal with, since it requires the knowledge of the carried traffic and thereby route blocking probabilities that can be computed in an exact way only for very small networks. A common feature of the methods to be applied is that they make use of the natural and well known reduced load and link independence assumptions, see [9], [10], [5]. Based on this approximation the partial derivatives of the network revenue can be found in a tractable form suitable for a gradient based hill climbing. Since this method has quite high running time, therefore, a somewhat simpler method based on a sequence of linear programming tasks is presented. Finally, a very simple alternative approach is suggested. All the three presented solution approaches assume that the ATM link capacities can take any real number, thus implying that the underlying cross connect structure is based on ATM.

\subsection{Gradient Based Hill Climbing}

Applying an extension of the arguments given in [8], see [2] for details, the partial derivatives of the network revenue can be computed. The computation requires that the fixed point equations associated with the reduced load approximation are solved, and in addition a set of linear equations has to be solved. The size of these equation systems equals to the product of the number of links in the network and the number of traffic types. 
Using these derivatives, the algorithmic solution is reduced for a standard gradient based hill climbing over a convex feasibility domain defined by linear inequalities. Here one can use either commercial or freely available software, see e.g. [3]. Unfortunately, however, the speed of the algorithm is greatly reduced by the large amount of side computations, mentioned in the preceding paragraph.

The algorithm will in general converge towards a local optimum. However, by a careful choice of the initial values, the risk of getting stuck in a local optimum that falls far from a global optimum can be reduced.

\subsection{Solution by Sequential Linear Programming}

In general, the complexity of computing blocking probabilities is much larger in the case where many different bandwidth demands (traffic types) co-exist. An efficient way to avoid the increased complexity is to approximate the blocking probability of a traffic type requiring $d$ units of capacity by grabbing $d$ times one unit independently. It is proven that this approximation asymptotically leads to the correct blocking probabilities, see [9] and [11].

Adopting this approximation and assuming all revenue coefficients are the same, independently of traffic types, it is shown in [4] that a slightly changed objective function and the same constraints can be accurately approximated by a sequence of linear programming tasks. The accuracy of the approximation increases in the regime where capacities are large.

In the following the total number of virtual links over all virtual networks is denoted by $J$, and the capacity of virtual link $j$ is denoted by $C_{j}$.

The incidence of physical and virtual links is expressed by a $K \times J$ zeroone structure matrix $\mathbf{S}$ ( $K$ is the number of physical links) in which the entries are given by

$$
s_{k, j}=\left\{\begin{array}{cc}
1 & \text { if physical link } k \text { is used by virtual link } j \\
0 & \text { otherwise }
\end{array}\right.
$$

Let us denote the blocking probability of logical link $j$ by $B_{j}$, and let $A_{i r}$ be the amount of capacity used by a route $r$ call on link $j$ and $\nu_{r}$ the offered traffic to route $r$.

$E(\rho, C)$ is Erlang's B-formula, and $a_{j}$ is a logarithmic blocking measure, defined by the procedure, related to link $j$. 
The algorithm is as follows, see [4] for details.

Step 1 Set $B_{j}:=0, a_{j}:=1$ for each $j$.

Step 2 Solve the linear programming problem

$$
\text { Maximize } \sum_{j} a_{j} C_{j}
$$

Subject to $S C \leq C_{\text {phys }}$ and $C \geq 0$.

( $C_{\text {phys }}$ is the physical capacity vector.)

Step 3 Compute new values for the blocking probabilities by

$$
B_{j}:=E\left(\left(1-B_{j}\right)^{-1} \sum_{r} A_{j r} \nu_{r} \prod_{i}\left(1-B_{i}\right)^{A_{i r}}, \tilde{C}_{j}\right)
$$

where $\tilde{C}_{1}, \ldots, \tilde{C}_{J}$ come from Step 2 as a solution of the linear programming problem.

Step 4 Set

$$
a_{j}:=-\log \left(1-B_{j}\right), \quad j=1, \ldots, J .
$$

Step 5 If all variables differ from their previous value by less then a given error treshold, then STOP, else repeat from Step 2.

The above iterative procedure has an intuitively appealing interpretation. If a link $j$ at a certain iteration has large blocking probability $B_{j}$, then the corresponding $a_{j}$ coefficient in the objective function will be large. That will inspire the linear programming to increase the value of $C_{j}$, since a variable with larger objective function coefficient can contribute more to the maximum. This conforms with the intuition that a link with high blocking probability needs capacity increment.

Numerical experience shows that the algorithm converges typically within a few iterations [4]. 


\subsection{Simplified Partitioning Based on Equivalent Link Blocking}

The approach to be presented here assumes that the traffic demand to each route is known in advance, implying that no optimization with respect to load sharing can take place. Besides having the traffic demand matrix as input, we also assume here that for each route $r$ a maximum allowed route bloking probability $B(r)$ is given.

Now some additional notations are introduced. Let $\mathcal{R}$ be the set of (fixed) routes in the network, taking all logical subnetworks into account. We want to design the logical link capacities such that the blocking probability of any route $r$ is at most $B(r)$.

Let $L(r)$ be the set of logical links used by route $r$ and denote by $l(r)$ the length of route $r$, that is, the number of links on the route.

Now, assuming link independence and using the reduced load approximation, the prescriptions on route blocking can clearly be satisfied if

$$
1-B_{j} \geq(1-B(r))^{1 / l(r)}
$$

holds for each route $r$ and for each logical link $j \in L(r)$. The idea here is that we distribute the route blocking probability evenly among the links used by the route. If $\mathcal{R}_{j}$ denotes the set of routes that use link $j$ then we obtain the condition

$$
1-B_{j} \geq \max _{r \in \mathcal{R}_{j}}(1-B(r))^{1 / l(r)}
$$

Now let $B_{j}^{0}$ be the maximum possible value for the blocking probability on link $j$ that follows from the above model, based on the idea of evenly distributed blocking. From the above considerations we have

$$
B_{j}^{0}=1-\max _{r \in \mathcal{R}_{j}}(1-B(r))^{1 / l(r)}
$$

Applying the $B_{j}^{0}$ values, we can approximate the link offered traffic as

$$
\rho_{j}^{0}=\sum_{r \in \mathcal{R}_{j}} A_{j r} \nu_{r} \prod_{i \neq j}\left(1-B_{i}^{0}\right)^{A_{i r}} .
$$

Once we know the value of $B_{j}^{0}$ and $\rho_{j}^{0}$ from the above explicite formulas, the capacity $C_{j}$ of logical link $j$ can now be calculated by inverting numerically Erlang's formula

$$
B_{j}^{0}=E\left(\rho_{j}^{0}, C_{j}\right)
$$


If the sum of the obtained $C_{j}$ logical capacity values exceed the available physical capacity on a physical link, then we normalize them such that they satisfy the physical capacity constraints. That is, we multiply all of them by a constant, smaller than 1 , such that their sum becomes equal to the physical capacity, while keeping the ratio of the logical capacities.

It should be noted that this procedure does not optimize the network revenue, it only dimensions the logical links, taking into account the requirements on route blocking probabilities. Thereby, it considers network revenue in an indirect way.

The computational complexity of this model is very small, since only the ATM-link capacities are varied and no linear programming is performed.

\section{Numerical Example}

The algorithms are being tested on various network examples. Due to space limitations, we show just very briefly the results of the comparison of two algorithms: the sequential linear programming approach, called Fixpoint and the equivalent link blocking approach, called ELB.

The two algorithms were compared on a 6 -node physical network that carried five different virtual ATM networks, each with four traffic classes. 16 different traffic scenarios were considered, characterized by two parameters. The parameter $\alpha$ defines the distribution of traffic among the classes. The higher the value of $\alpha$, the more unbalanced is the distribution. The parameter $\beta$ defines the homogenity of bandwidth demands: the higher the value of $\beta$, the more inhomogeneous are the bandwidth demands.

The results are shown in Fig. 3. For balanced and homogeneous traffic scenarios the two methods show the same performance. For unbalanced and/or inhomogeneous traffic demands, however, the more sophisticated Fixpoint algorithm performs significantly better, according to the expectations.

On the other hand, when running time is considered, we find a converse relationship, see Fig. 4. Although for very small networks there is no significant difference in running times, but if the network size gets larger, such as a 6 -node physical network with 5 virtual subnetworks carriyng 4 traffic classes, as in the example, then there is already a dramatic difference in running times. This shows a clear trade-off between quality and speed: if rougher estimates suffice, then ELB is recommended. On the other hand, if more 
accuracy is needed, then the slower but more accurate Fixpoint algorithm is favourable.

\section{Conclusion}

A framework and model, along with efficient solution algorithms, are presented to dimension virtual ATM networks on top of the same physical infrastructure network, such that the virtual networks share the infrastructure, while the total network revenue is optimized. The algorithms are tried on various network scenarios and a trade-off between the quality of the result and running time is exhibited. The algorithms are being implemented as part of an integrated system for ATM network planning, simulation and management called PLASMA [6].

\section{Acknowledgment}

The research was performed as part of a joint project between Ellemtel Telecommunication System Laboratories, Sweden and the Department of Telecommunications and Telematics, Technical University of Budapest, Hungary. The authors are grateful to Miklós Boda and Géza Gordos for stimulating discussions and for their continuous support.

\section{References}

[1] A. Arvidsson, Management of Reconfigurable Virtual Path Networks, ITC-14, France, 1994, pp. 931-940.

[2] A. Faragó, S. Blaabjerg, L. Ast, G. Gordos and T. Henk, "A New Degree of Freedom in ATM Network Dimensioning: Optimizing the Logical Configuration", to appear in IEEE Journal on Selected Areas of Communications, Spec. Issue on the Fundamentals of Networking.

[3] C. Lawrence J.L. Zhou, A.L. Tits, "Users Guide for CFSQP version 2.2: A C Code for Solving (Large Scale) Constrained Nonlinear (Minimax) Optimization Problems, Generating Iterates Satisfying All Inequality 
Constraints", E.E. Department, Institute for System Research, University of Maryland, TR 94-16r1.

[4] A. Faragó, S. Blaabjerg, W. Holender, T. Henk, L. Ast, Á. Szentesi, Zs. Ziaja, "Resource Separation - an Efficient Tool for Optimizing ATM Network Configuration", Proc. NETWORKS'94, Sixth International Network Planning Symp., Budapest, Hungary, Sept. 1994, pp. 83-88.

[5] A. Girard, Routing and Dimensioning in Circuit Switched Networks, Addison Wesley, 1990.

[6] Zs. Haraszti, I. Dahlqvist, A. Faragó and T. Henk, "PLASMA - An Integrated Tool for ATM Network Operation", International Switching Symp. (ISS'95), Stockholm, 1995.

[7] T. Hadoung, B. Stavenow, J. Dejean, "The Stratified Reference Model: An Open Architecture to B-ISDN", International Switching Symp. (ISS'90), Stockholm, 1990.

[8] F.P. Kelly, "Loss Networks", The Annals of Applied Probability, 1(1991/3), pp. 319-378.

[9] F.P. Kelly, "Blocking Probabilities in Large Circuit Switched Networks", Adv. Appl. Probab., 18(1986), pp. 473-505.

[10] F.P. Kelly, "Routing in Circuit Switched Networks: Optimization, Shadow Prices and Decentralization", Adv. Appl. Probab., 20(1988), pp. 112-144.

[11] J.-F. P. Labourdette and G.W. Hart, "Blocking Probabilities in Multitraffic Loss Systems: Insensitivity, Asymptotic Behavior and Approximations, IEEE Trans. Communications, 40(1992/8) pp. 1355-1366.

[12] M. Menozzi, U. Mocci, C. Scoglio, A. Tonietti, Traffic Integration and Virtual Path Optimization in ATM Networks, Networks'94, pp. 71-76.

[13] M. Ritter, P. Tran-Gia (Eds.), Multi-Rate Models for Dimensioning and Performance Evaluation of ATM Networks, COST 242 Interim Report, Commission of the European Communities, June 1994. 


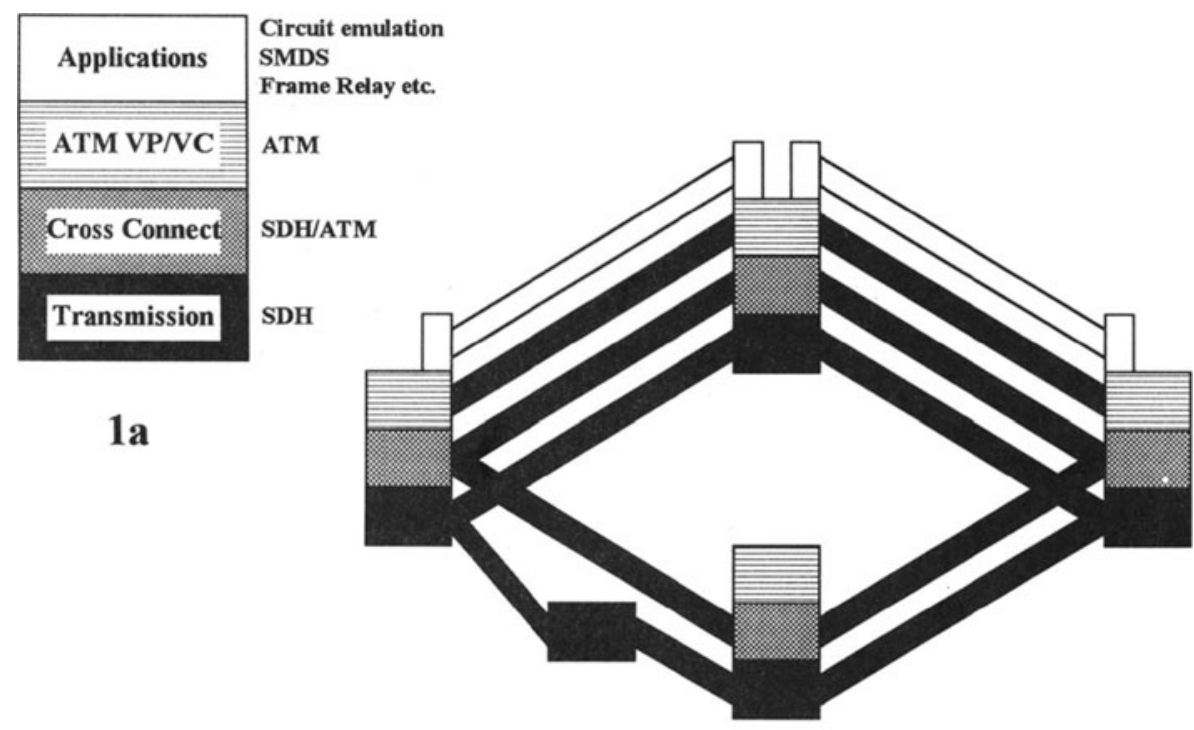

1b

Fig. $1 \mathrm{~B}-I S D N$ from the viewpoint of the stratified reference model 1a) the protocol viewpoint, 1b) the network viewpoint

\begin{tabular}{|c|c|c|c|}
\hline \multicolumn{2}{|c|}{ ATM } & \multicolumn{2}{c|}{ ATM } \\
1 & \multicolumn{2}{c|}{2} \\
\hline STM & STM & STM & STM \\
1 & 1 & 1 & 1 \\
\hline \multicolumn{3}{|c|}{ STM-4 } \\
\hline
\end{tabular}

$2 a$
ATM VP/VC

Cross Connect

$\mathrm{SDH}$

Transmission

SDH

\begin{tabular}{|c|c|c|}
\hline $\begin{array}{c}\text { ATM } \\
1\end{array}$ & \multicolumn{2}{|c|}{ ATM } \\
\hline VP1 & VP2 & VP3 \\
\hline \multicolumn{2}{|c|}{ STM-4 } \\
\hline
\end{tabular}

ATM VP/VC

Cross Connect

ATM VP

Transmission

SDH

Fig. 2 Partitioning of the Cross Connect infrastructure. In case where it is based on SDH whole blocks of STM-1 must be chosen, while in case of ATM VPS any continuous values are feasible. 

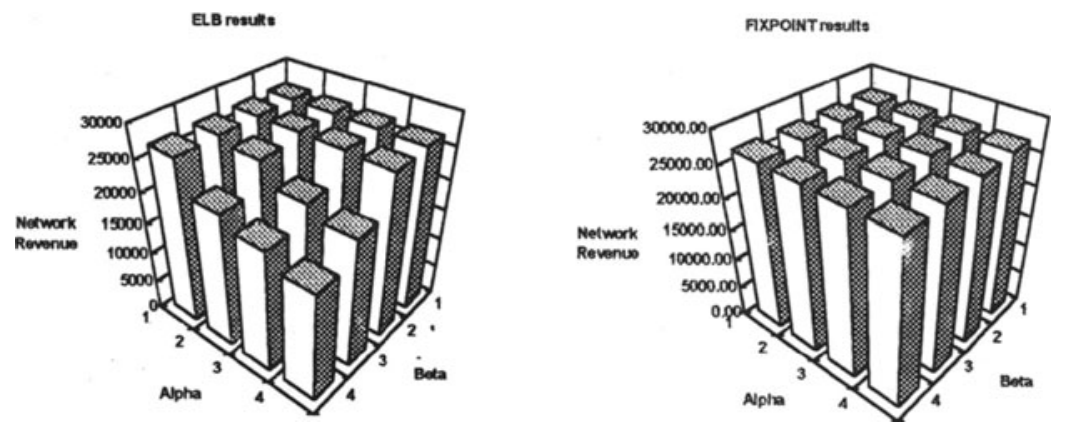

Fig. 3 Comparison of total network revenue

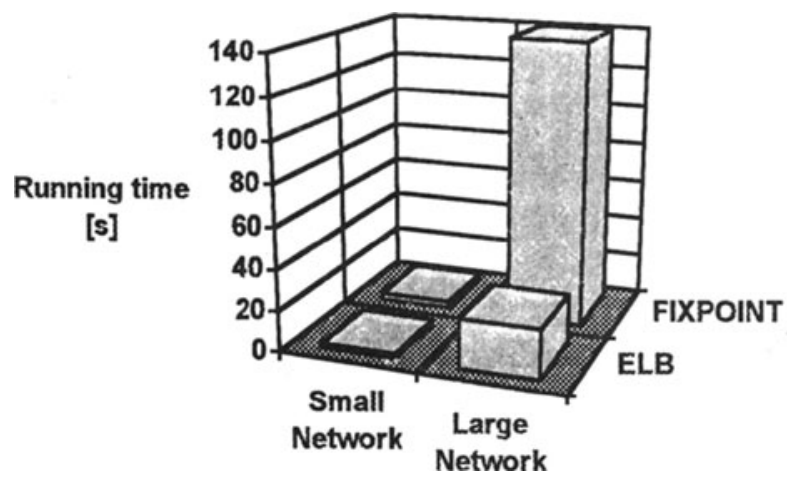

Fig. 4 Comparison of running times 